\title{
AN ANALYTICAL APPROACH FOR THE GREEN'S FUNCTIONS OF BIHARMONIC PROBLEMS WITH CIRCULAR AND ANNULAR DOMAINS
}

\author{
J. T. Chen ${ }^{*}$ H. Z. Liao ${ }^{* *}$ \\ Department of Harbor and River Engineering \\ Department of Mechanical and Mechatronic Engineering \\ National Taiwan Ocean University \\ Keelung, Taiwan 20224, R.O.C. \\ W. M. Lee ${ }^{* * *}$ \\ Department of Mechanical Engineering \\ China Institute of Technology \\ Taipei, Taiwan 11581, R.O.C.
}

\begin{abstract}
In this paper, an analytical approach for deriving the Green’s function of circular and annular plate was presented. Null-field integral equations were employed to solve the plate problems while kernel functions were expanded to degenerate kernels. The unknown boundary data of the displacement, slope, normal moment and effective shear force were expressed in terms of Fourier series. It was noticed that all the improper integrals were avoided when the degenerate kernels were used. After determining the unknown Fourier coefficients, the displacement, slope, normal moment and effective shear force of the plate could be obtained by using the boundary integral equations. The present approach was seen as an "analytical” approach for a series solution. Finally, several analytical solutions were obtained. To see the validity of the present method, FEM solutions using ABAQUS were compared well with our analytical solutions. The displacement, radial moment and shear variations of radial and angular positions were presented.
\end{abstract}

Keywords : Green's function, Null-field integral equation, Degenerate kernel, Fourier series.

\section{INTRODUCTION}

Green's functions play an important role in solving numerous problems in mechanics. It is the basic tool of many numerical and analytical techniques, e.g., boundary element methods and integral equation methods. Green's functions were widely used for the qualitative analysis of initial and boundary value problems for equations of all the standard (elliptic, parabolic and hyperbolic) types in mathematical physics. Boley proposed an iterated form to obtain the analytical Green's function [1]. Wang and Sudak [2] derived two-dimensional anti-plane time-harmonic Green's functions for a circular inclusion with an imperfect interface. Time-harmonic Green's functions can be applied to formulate the boundary integral equations for the time-harmonic problems, and can also be employed to investigate the dynamic Eshelby problem and scattering problems in elastodynamics. Several techniques had earlier been developed and could be successfully utilized for computing Green's functions for a certain class of multiply-connected regions. Timoshenko and Woinowsky-Krieger [3] also treated the circular plate problems in their books. In the Melnikov's paper [4], a semi-analytical approach was applied to construct Green's functions and matrices of Green's type for the Laplace and Klein-Gordon equations in two dimensions. Mixed boundary value problems posed in multiplyconnected regions were also concerned by Melnikov [5]. Melnikov [6] also investigated the Green's function of plate problems. A Green's function of the biharmonic equation that can model bending of a thin plate is usually referred to as its influence function in elasticity. Recently, Sharafutdinov [7] had considered the problems of an annular plate subjected to a concentrated load along its edges using functions of a complex variable. In the recent years, the Green's function of an isotropic annular plate clamped along one edge and free at the other was solved by Adewale [8]. Following the success of null-field integral formulation for typical BVPs [9-11], the present paper provides an analytical approach by using null-field integral equations in conjunction with Fourier series and degenerate kernels to derive the Green's functions of circular and annular plates.

\footnotetext{
* Life-time Distinguished Professor $\quad * *$ Master student $\quad * * *$ Associate Professor
} 


\section{A UNIFIED FORMULATION FOR THE \\ GREEN'S FUNCTION USING NULL-FIELD INTEGRAL EQUATION APPROACH}

\subsection{Problem Statement for a Thin Plate}

Considering a Kirchhoff plate for the twodimensional domain under the concentrated load, the governing equation is written as follows:

$$
\nabla^{4} G(x, \zeta)=\frac{\delta(x-\zeta)}{D}, \quad x \in \Omega,
$$

where $G(x, \zeta)$ is the Green's function and can be seen as the displacement at $x$ due to a concentrated load at $\zeta$, $\delta(x-\zeta)$ denotes the Dirac-delta function of source at $\zeta$, $\Omega$ is the domain of the thin plate and $D$ is the flexural rigidity of the plate which is expressed as

$$
D=\frac{E h^{3}}{12\left(1-\mu^{2}\right)},
$$

in which $E$ is the Young's modulus, $\mu$ denotes the Poisson ratio, and $h$ is the plate thickness. In order to employ the Rayleigh-Green identity [9], we need two systems, $u(x)$ and $v(x)$. We choose $G(x, \zeta)$ as $u(x)$ and the fundamental solution $U(s, x)$ as $v(x)$. The fundamental solution satisfies

$$
\nabla^{4} U(s, x)=8 \pi \delta(s-x)
$$

where

$$
U(s, x)=r^{2} \ln r
$$

in which $r$ is the distance between the source point $s$ and the field point $x(r \equiv|x-s|)$. After exchanging with the variables $x$ and $s$, we have four boundary integral equations as shown in the next section.

\subsection{Conventional and Present Null-Field Boundary Integral Formulations}

The conventional boundary integral equations for the domain point can be derived from the Rayleigh-Green identity [9] as shown below:

$$
\begin{aligned}
8 \pi G(\zeta, x)= & -\int_{B} U(s, x) K_{v, s}[G(s, \zeta)] d B(s) \\
& +\int_{B} \Theta(s, x) K_{m, s}[G(s, \zeta)] d B(s) \\
& -\int_{B} M(s, x) K_{\theta, s}[G(s, \zeta)] d B(s) \\
& +\int_{B} V(s, x) G(s, \zeta) d B(s) \\
& +U(\zeta, x), \quad x \in \Omega
\end{aligned}
$$

$$
\begin{aligned}
8 \pi K_{\theta, x}[G(\zeta, x)]= & -\int_{B} U_{\theta}(s, x) K_{v, s}[G(s, \zeta)] d B(s) \\
& +\int_{B} \Theta_{\theta}(s, x) K_{m, s}[G(s, \zeta)] d B(s) \\
& -\int_{B}^{B} M_{\theta}(s, x) K_{\theta, s}[G(s, \zeta)] d B(s) \\
& +\int_{B} V_{\theta}(s, x) G(s, \zeta) d B(s) \\
& +U_{\theta}(\zeta, x), \quad x \in \Omega
\end{aligned}
$$

$$
\begin{aligned}
8 \pi K_{m, x}[G(\zeta, x)]= & -\int_{B} U_{m}(s, x) K_{v, s}[G(s, \zeta)] d B(s) \\
& +\int_{B} \Theta_{m}(s, x) K_{m, s}[G(s, \zeta)] d B(s) \\
& -\int_{B} M_{m}(s, x) K_{\theta, s}[G(s, \zeta)] d B(s) \\
& +\int_{B} V_{m}(s, x) G(s, \zeta) d B(s) \\
& +U_{m}(\zeta, x), \quad x \in \Omega
\end{aligned}
$$

$$
\begin{aligned}
8 \pi K_{v, x}[G(\zeta, x)]= & -\int_{B} U_{v}(s, x) K_{v, s}[G(s, \zeta)] d B(s) \\
& +\int_{B} \Theta_{v}(s, x) K_{m, s}[G(s, \zeta)] d B(s) \\
& -\int_{B}^{B} M_{v}(s, x) K_{\theta, s}[G(s, \zeta)] d B(s) \\
& +\int_{B} V_{v}(s, x) G(s, \zeta) d B(s) \\
& +U_{v}(\zeta, x), \quad x \in \Omega
\end{aligned}
$$

where $B$ is the boundary of the domain $\Omega, G(x, \zeta)$, $K_{\theta, x}[G(x, \zeta)], K_{m, x}[G(x, \zeta)]$ and $K_{v, x}[G(x, \zeta)]$ are the displacement, slope, normal moment and effective shear force, respectively. The kernel functions $U, \Theta, M, V, U_{\theta}$, $\Theta_{\theta}, M_{\theta}, V_{\theta}, U_{m}, \Theta_{m}, M_{m}, V_{m}, U_{v}, \Theta_{v}, M_{v}$ and $V_{v}$ in Eqs. (5) $\sim$ (8) will be elaborated on later. The $K_{\theta, x}[\cdot], K_{m, x}[\cdot]$ and $K_{v, x}[\cdot]$ are the slope, moment and shear force operators with respect to the displacement field at point $x$ and are defined as follows:

$$
K_{\theta, x}[\cdot]=\frac{\partial}{\partial n_{x}},
$$

$$
\begin{gathered}
K_{m, x}[\cdot]=v \nabla_{x}^{2}+(1-v) \frac{\partial^{2}}{\partial^{2} n_{x}}, \\
K_{v, x}[\cdot]=\frac{\partial \nabla_{x}^{2}}{\partial n_{x}}+(1-v) \frac{\partial}{\partial t_{x}}\left[\frac{\partial}{\partial n_{x}}\left(\frac{\partial}{\partial t_{x}}\right)\right],
\end{gathered}
$$

where $\partial / \partial n_{x}$ is the normal derivative with respect to the field point $x, \partial / \partial t_{x}$ is the tangential derivative with respect to the field point $x$, and $\nabla_{x}^{2}$ means the Laplacian operator. By moving the field point to the boundary, Eqs. (5) (8) are reduced to

$$
\begin{aligned}
4 \pi G(x, \zeta)= & -R P V \cdot \int_{B} U(s, x) K_{v, s}[G(s, \zeta)] d B(s) \\
& +R P V \cdot \int_{B} \Theta(s, x) K_{m, s}[G(s, \zeta)] d B(s) \\
& -R P V \cdot \int_{B}^{B} M(s, x) K_{\theta, s}[G(s, \zeta)] d B(s) \\
& +C P V \cdot \int_{B} V(s, x) G(s, \zeta) d B(s) \\
& +U(\zeta, x), \quad x \in B
\end{aligned}
$$




$$
\begin{aligned}
4 \pi K_{\theta, x}[G(x, \zeta)]= & -R P V \cdot \int_{B} U_{\theta}(s, x) K_{v, s}[G(s, \zeta)] d B(s) \\
& +R P V \cdot \int_{B} \Theta_{\theta}(s, x) K_{m, s}[G(s, \zeta)] d B(s) \\
& -C P V \cdot \int_{B}^{B} M_{\theta}(s, x) K_{\theta, s}[G(s, \zeta)] d B(s) \\
& +H P V \cdot \int_{B} V_{\theta}(s, x) G(s, \zeta) d B(s) \\
& +U_{\theta}(\zeta, x), \quad x \in B
\end{aligned}
$$

$$
\begin{aligned}
4 \pi K_{m, x}[G(x, \zeta)]= & -R P V \cdot \int_{B} U_{m}(s, x) K_{v, s}[G(s, \zeta)] d B(s) \\
& +C P V \cdot \int_{B} \Theta_{m}(s, x) K_{m, s}[G(s, \zeta)] d B(s) \\
& -H P V \cdot \int_{B} M_{m}(s, x) K_{\theta, s}[G(s, \zeta)] d B(s) \\
& +F P \cdot \int_{B} V_{m}(s, x) G(s, \zeta) d B(s) \\
& +U_{m}(\zeta, x), \quad x \in B
\end{aligned}
$$

$4 \pi K_{v, x}[G(x, \zeta)]=-C P V \cdot \int_{B} U_{v}(s, x) K_{v, s}[G(s, \zeta)] d B(s)$

$$
\begin{aligned}
& +H P V \cdot \int_{B} \Theta_{v}(s, x) K_{m, s}[G(s, \zeta)] d B(s) \\
& -F P \cdot \int_{B} M_{v}(s, x) K_{\theta, s}[G(s, \zeta)] d B(s) \\
& +F P \cdot \int_{B} V_{v}(s, x) G(s, \zeta) d B(s) \\
& +U_{v}(\zeta, x), \quad x \in B
\end{aligned}
$$

where $C P V, R P V, H P V$ and $F P$ denote the Cauchy principal value, Riemann principal value, Hadamard principal value and the finite part [10], respectively. The conventional null-field integral equations by moving the field point $x$ outside the domain are shown below:

$$
\begin{aligned}
0= & -\int_{B} U(s, x) K_{v, s}[G(s, \zeta)] d B(s) \\
& +\int_{B} \Theta(s, x) K_{m, s}[G(s, \zeta)] d B(s) \\
& -\int_{B}^{B} M(s, x) K_{\theta, s}[G(s, \zeta)] d B(s) \\
& +\int_{B} V(s, x) G(s, \zeta) d B(s)+U(\zeta, x), \quad x \in \Omega^{c}
\end{aligned}
$$

$$
\begin{aligned}
0= & -\int_{B} U_{\theta}(s, x) K_{v, s}[G(s, \zeta)] d B(s) \\
& +\int_{B}^{B} \Theta_{\theta}(s, x) K_{m, s}[G(s, \zeta)] d B(s) \\
& -\int_{B}^{B} M_{\theta}(s, x) K_{\theta, s}[G(s, \zeta)] d B(s) \\
& +\int_{B} V_{\theta}(s, x) G(s, \zeta) d B(s)+U_{\theta}(\zeta, x), \quad x \in \Omega^{c}(
\end{aligned}
$$

$$
\begin{aligned}
0= & -\int_{B} U_{m}(s, x) K_{v, s}[G(s, \zeta)] d B(s) \\
& +\int_{B} \Theta_{m}(s, x) K_{m, s}[G(s, \zeta)] d B(s) \\
& -\int_{B} M_{m}(s, x) K_{\theta, s}[G(s, \zeta)] d B(s) \\
& +\int_{B} V_{m}(s, x) G(s, \zeta) d B(s)+U_{m}(\zeta, x), \quad x \in \Omega^{c}
\end{aligned}
$$

$$
\begin{aligned}
0= & -\int_{B} U_{v}(s, x) K_{v, s}[G(s, \zeta)] d B(s) \\
& +\int_{B} \Theta_{v}(s, x) K_{m, s}[G(s, \zeta)] d B(s) \\
& -\int_{B}^{B} M_{v}(s, x) K_{\theta, s}[G(s, \zeta)] d B(s) \\
& +\int_{B} V_{v}(s, x) G(s, \zeta) d B(s)+U_{v}(\zeta, x), \quad x \in \Omega^{c}
\end{aligned}
$$

where $\Omega^{C}$ is the complementary domain of $\Omega$. If the kernel functions in Eqs. (5) (8) and (16) (19) are expressed by using the degenerate (separable) forms for $\Omega$ or $\Omega^{C}$ domain, we have alternative representation as shown below:

$$
\begin{aligned}
8 \pi G(\zeta, x)= & -\int_{B} U(s, x) K_{v, s}[G(s, \zeta)] d B(s) \\
& +\int_{B} \Theta(s, x) K_{m, s}[G(s, \zeta)] d B(s) \\
& -\int_{B} M(s, x) K_{\theta, s}[G(s, \zeta)] d B(s) \\
& +\int_{B} V(s, x) G(s, \zeta) d B(s) \\
& +U(\zeta, x), \quad x \in \Omega \cup B \\
8 \pi K_{\theta, x}[G(\zeta, x)]= & -\int_{B} U_{\theta}(s, x) K_{v, s}[G(s, \zeta)] d B(s) \\
& +\int_{B} \Theta_{\theta}(s, x) K_{m, s}[G(s, \zeta)] d B(s) \\
& -\int_{B}^{B} M_{\theta}(s, x) K_{\theta, s}[G(s, \zeta)] d B(s) \\
& +\int_{B}^{B} V_{\theta}(s, x) G(s, \zeta) d B(s) \\
& +U_{\theta}(\zeta, x), \quad x \in \Omega \cup B
\end{aligned}
$$

$8 \pi K_{m, x}[G(\zeta, x)]=-\int_{B} U_{m}(s, x) K_{v, s}[G(s, \zeta)] d B(s)$

$$
\begin{aligned}
& +\int_{B} \Theta_{m}(s, x) K_{m, s}[G(s, \zeta)] d B(s) \\
& -\int_{B}^{B} M_{m}(s, x) K_{\theta, s}[G(s, \zeta)] d B(s) \\
& +\int_{B} V_{m}(s, x) G(s, \zeta) d B(s) \\
& +U_{m}(\zeta, x), \quad x \in \Omega \cup B
\end{aligned}
$$

$$
\begin{aligned}
8 \pi K_{v, x}[G(\zeta, x)]= & -\int_{B} U_{v}(s, x) K_{v, s}[G(s, \zeta)] d B(s) \\
& +\int_{B} \Theta_{v}(s, x) K_{m, s}[G(s, \zeta)] d B(s) \\
& -\int_{B}^{B} M_{v}(s, x) K_{\theta, s}[G(s, \zeta)] d B(s) \\
& +\int_{B} V_{v}(s, x) G(s, \zeta) d B(s) \\
& +U_{v}(\zeta, x), \quad x \in \Omega \cup B
\end{aligned}
$$

$$
\begin{aligned}
0= & -\int_{B} U(s, x) K_{v, s}[G(s, \zeta)] d B(s) \\
& +\int_{B} \Theta(s, x) K_{m, s}[G(s, \zeta)] d B(s) \\
& -\int_{B}^{B} M(s, x) K_{\theta, s}[G(s, \zeta)] d B(s) \\
& +\int_{B}^{B} V(s, x) G(s, \zeta) d B(s) \\
& +U(\zeta, x), \quad x \in \Omega^{c} \cup B
\end{aligned}
$$




$$
\begin{aligned}
0= & -\int_{B} U_{\theta}(s, x) K_{v, s}[G(s, \zeta)] d B(s) \\
& +\int_{B} \Theta_{\theta}(s, x) K_{m, s}[G(s, \zeta)] d B(s) \\
& -\int_{B} M_{\theta}(s, x) K_{\theta, s}[G(s, \zeta)] d B(s) \\
& +\int_{B}^{B} V_{\theta}(s, x) G(s, \zeta) d B(s) \\
& +U_{\theta}(\zeta, x), x \in \Omega^{c} \cup B \\
0= & -\int_{B} U_{m}(s, x) K_{v, s}[G(s, \zeta)] d B(s) \\
& +\int_{B} \Theta_{m}(s, x) K_{m, s}[G(s, \zeta)] d B(s) \\
& -\int_{B} M_{m}(s, x) K_{\theta, s}[G(s, \zeta)] d B(s) \\
& +\int_{B} V_{m}(s, x) G(s, \zeta) d B(s) \\
& +U_{m}(\zeta, x), \quad x \in \Omega^{c} \cup B \\
0= & -\int_{B} U_{v}(s, x) K_{v, s}[G(s, \zeta)] d B(s) \\
& +\int_{B}^{B} \Theta_{v}(s, x) K_{m, s}[G(s, \zeta)] d B(s) \\
& -\int_{B}^{B} M_{v}(s, x) K_{\theta, s}[G(s, \zeta)] d B(s) \\
& +\int_{B} V_{v}(s, x) G(s, \zeta) d B(s) \\
& +U_{v}(\zeta, x), x \in \Omega^{c} \cup B
\end{aligned}
$$

It is noted that the collocation position of Eqs. (20) (27) can include the boundary point. Although Eqs. (24) (27) in the plate formulation are provided, only the first two equations are employed to solve unknown boundary data for simplicity. In the real implementation, the collocation point in the null-field integral equation can be exactly located on the real boundary from $\Omega^{C}$ once the kernel functions is expressed in terms of interior and exterior appropriate forms of degenerate kernels. In other words, Eqs. (24) (27) are implemented for $x \in \Omega^{C} \cup B$ if kernels of degenerate forms are properly used in the Wu's thesis [15]. Consequently, all the improper integrals disappear in the BIEs since the potential across the boundary can be determined in both sides by using the degenerate kernels [16].

\subsection{Expansion of Kernels}

For the kernel function $U(s, x)$, it can be expanded in terms of degenerate kernel in a series form as shown below:

$$
U(s, x)=\left\{\begin{aligned}
U^{I}(s, x)= & \rho^{2}(1+\ln R)+R^{2} \ln R-\left[R \rho(1+2 \ln R)+\frac{1}{2} \frac{\rho^{3}}{R}\right] \cos (\theta-\phi) \\
& -\sum_{m=2}^{\infty}\left[\frac{1}{m(m+1)} \frac{\rho^{m+2}}{R^{m}}-\frac{1}{m(m-1)} \frac{\rho^{m}}{R^{m-2}}\right] \cos [m(\theta-\phi)], \quad R \geq \rho \\
U^{E}(s, x)= & R^{2}(1+\ln \rho)+\rho^{2} \ln \rho-\left[\rho R(1+2 \ln \rho)+\frac{1}{2} \frac{R^{3}}{\rho}\right] \cos (\theta-\phi) \\
& -\sum_{m=2}^{\infty}\left[\frac{1}{m(m+1)} \frac{R^{m+2}}{\rho^{m}}-\frac{1}{m(m-1)} \frac{R^{m}}{\rho^{m-2}}\right] \cos [m(\theta-\phi)], \quad \rho>R
\end{aligned}\right.
$$

where $x=(\rho, \phi), s=(R, \theta)$, the superscripts " $I$ " and " $E$ " denote the interior and exterior cases of $U(s, x)$ kernel depending on the geometry as shown in Fig. 1. Then, the kernel function with the superscript " $I$ " is chosen while the field point is inside the circular region; otherwise, the kernel with the superscript " $E$ " is chosen. The other degenerate kernels in the boundary integral equations can be obtained by utilizing the operators of Eqs. (9) (11) with respect to the $U(s, x)$ kernel. The degenerate kernels of $U, \Theta, M, V, U_{\theta}, \Theta_{\theta}, M_{\theta}, V_{\theta}, U_{m}, \Theta_{m}, M_{m}, V_{m}, U_{v}$, $\Theta_{v}, M_{v}$ and $V_{v}$ in Eqs. (20) (23) are listed in [9-11].

\section{SERIES REPRESENTATION FOR THE GREEN'S FUNCTION OF THE ANNULAR PLATE}

For the annular case as shown in Fig. 2, the unknown Fourier coefficients can be analytically determined. By collocating $x$ on $\left(b^{+}, \phi\right)$ and $\left(a^{-}, \phi\right)$, Eqs. (24) and (25) yield

$$
\begin{aligned}
0= & -\int_{B_{1}} U^{E}(s, x) K_{v, s}[G(s, \zeta)] d B(s) \\
& +\int_{B_{1}} \Theta^{E}(s, x) K_{m, s}[G(s, \zeta)] d B(s) \\
& -\int_{B_{1}} M^{E}(s, x) K_{\theta, s}[G(s, \zeta)] d B(s) \\
& +\int_{B_{1}} V^{E}(s, x) G(s, \zeta) d B(s) \\
& -\int_{B_{2}} U^{E}(s, x) K_{v, s}[G(s, \zeta)] d B(s) \\
& +\int_{B_{2}} \Theta^{E}(s, x) K_{m, s}[G(s, \zeta)] d B(s) \\
& -\int_{B_{2}} M^{E}(s, x) K_{\theta, s}[G(s, \zeta)] d B(s) \\
& +\int_{B_{2}} V^{E}(s, x) G(s, \zeta) d B(s)+U^{E}(\zeta, x), \quad \rho \in b^{+},
\end{aligned}
$$




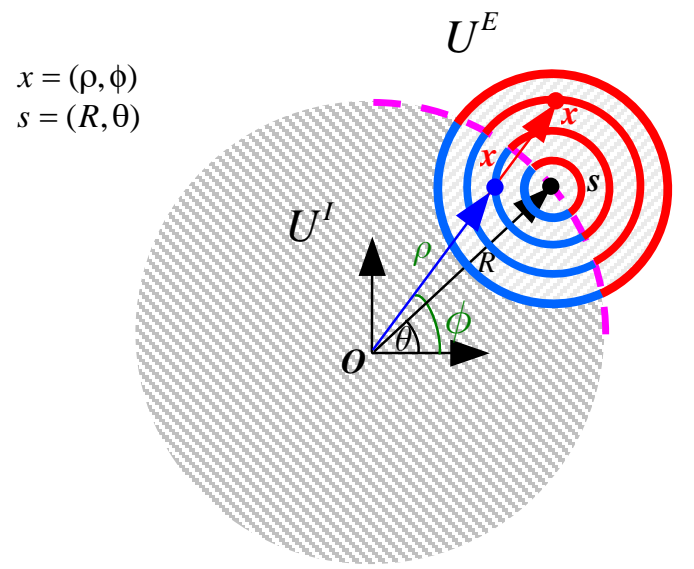

Fig. 1 Degenerate kernel for $U(s, x)$

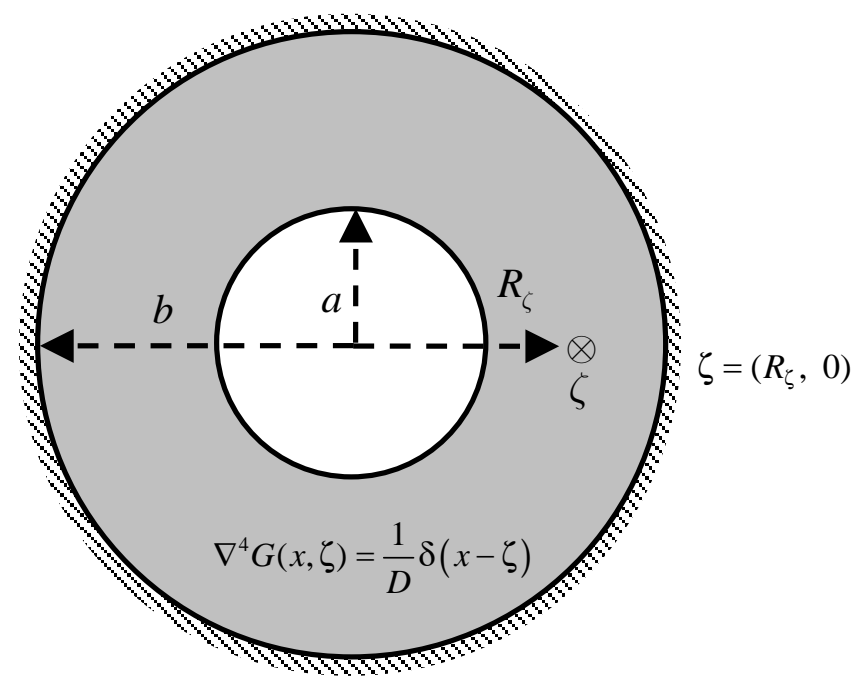

Fig. 2 Green's function of the biharmonic equation for the annular plate problem $\left(R_{\zeta}\right.$ is the distance between the source and the center of the circle)

$$
\begin{aligned}
0= & -\int_{B_{1}} U_{\theta}^{E}(s, x) K_{v, s}[G(s, \zeta)] d B(s) \\
& +\int_{B_{1}} \Theta_{\theta}^{E}(s, x) K_{m, s}[G(s, \zeta)] d B(s) \\
& -\int_{B_{1}} M_{\theta}^{E}(s, x) K_{\theta, s}[G(s, \zeta)] d B(s) \\
& +\int_{B_{1}} V_{\theta}^{E}(s, x) G(s, \zeta) d B(s) \\
& -\int_{B_{2}} U_{\theta}^{E}(s, x) K_{v, s}[G(s, \zeta)] d B(s) \\
& +\int_{B_{2}} \Theta_{\theta}^{E}(s, x) K_{m, s}[G(s, \zeta)] d B(s) \\
& -\int_{B_{2}} M_{\theta}^{E}(s, x) K_{\theta, s}[G(s, \zeta)] d B(s) \\
& +\int_{B_{2}} V_{\theta}^{E}(s, x) G(s, \zeta) d B(s)+U_{\theta}^{E}(\zeta, x), \quad \rho \in b^{+},
\end{aligned}
$$

$$
\begin{aligned}
& 0=-\int_{B_{1}} U^{I}(s, x) K_{v, s}[G(s, \zeta)] d B(s) \\
& +\int_{B_{1}} \Theta^{I}(s, x) K_{m, s}[G(s, \zeta)] d B(s) \\
& -\int_{B_{1}} M^{I}(s, x) K_{\theta, s}[G(s, \zeta)] d B(s) \\
& +\int_{B_{1}} V^{I}(s, x) G(s, \zeta) d B(s) \\
& -\int_{B_{2}} U^{I}(s, x) K_{v, s}[G(s, \zeta)] d B(s) \\
& +\int_{B_{2}} \Theta^{I}(s, x) K_{m, s}[G(s, \zeta)] d B(s) \\
& -\int_{B_{2}} M^{I}(s, x) K_{\theta, s}[G(s, \zeta)] d B(s) \\
& +\int_{B_{2}} V^{I}(s, x) G(s, \zeta) d B(s)+U^{I}(\zeta, x), \quad \rho \in a^{-} \text {, }
\end{aligned}
$$

$$
\begin{aligned}
0= & -\int_{B_{1}} U_{\theta}^{I}(s, x) K_{v, s}[G(s, \zeta)] d B(s) \\
& +\int_{B_{1}} \Theta_{\theta}^{I}(s, x) K_{m, s}[G(s, \zeta)] d B(s) \\
& -\int_{B_{1}} M_{\theta}^{I}(s, x) K_{\theta, s}[G(s, \zeta)] d B(s) \\
& +\int_{B_{1}} V_{\theta}^{I}(s, x) G(s, \zeta) d B(s) \\
& -\int_{B_{2}} U_{\theta}^{I}(s, x) K_{v, s}[G(s, \zeta)] d B(s) \\
& +\int_{B_{2}} \Theta_{\theta}^{I}(s, x) K_{m, s}[G(s, \zeta)] d B(s) \\
& -\int_{B_{2}} M_{\theta}^{I}(s, x) K_{\theta, s}[G(s, \zeta)] d B(s) \\
& +\int_{B_{2}} V_{\theta}^{I}(s, x) G(s, \zeta) d B(s)+U_{\theta}^{I}(\zeta, x), \quad \rho \in a^{-},
\end{aligned}
$$

The Fourier expansions of the boundary densities for the radial slope, normal moment and shear force are shown below:

$$
K_{v, s}[G(s, \zeta)]=a_{0}+\sum_{n=1}^{M}\left(a_{n} \cos n \theta+b_{n} \sin n \theta\right), \quad s \in B,
$$

$K_{m, s}[G(s, \zeta)]=\bar{a}_{0}+\sum_{n=1}^{M}\left(\bar{a}_{n} \cos n \theta+\bar{b}_{n} \sin n \theta\right), \quad s \in B$,

$K_{\theta, s}[G(s, \zeta)]=p_{0}+\sum_{n=1}^{M}\left(p_{n} \cos n \theta+q_{n} \sin n \theta\right), \quad s \in B$,

$G(s, \zeta)=\bar{p}_{0}+\sum_{n=1}^{M}\left(\bar{p}_{n} \cos n \theta+\bar{q}_{n} \sin n \theta\right), \quad s \in B$,

where $a_{n}, b_{n}, \bar{a}_{n}, \bar{b}_{n}, p_{n}, q_{n}, \bar{p}_{n}$ and $\bar{q}_{n}(n=0,1,2 \cdots)$ are the Fourier coefficients. By substituting all the boundary data into Eqs. (29) (32) for the direct problem, we obtain the unknown Fourier coefficients after comparing with the coefficients of constant, $\cos \left(\theta_{\zeta}-\theta\right)$ and $\cos n\left(\theta_{\zeta}-\theta\right)$ terms. By substituting all the boundary densities into the integral representation for the domain point, we have the Green's function as shown below: 


$$
\begin{aligned}
8 \pi G(x, \zeta)= & -\int_{B_{1}} U^{I}(s, x) K_{v, s}[G(s, \zeta)] d B(s) \\
& +\int_{B_{1}} \Theta^{I}(s, x) K_{m, s}[G(s, \zeta)] d B(s) \\
& -\int_{B_{1}} M^{I}(s, x) K_{\theta, s}[G(s, \zeta)] d B(s) \\
& +\int_{B_{1}}^{V_{1}} V^{I}(s, x) G(s, \zeta) d B(s) \\
& -\int_{B_{2}}^{U^{E}}(s, x) K_{v, s}[G(s, \zeta)] d B(s) \\
& +\int_{B_{2}} \Theta^{E}(s, x) K_{m, s}[G(s, \zeta)] d B(s) \\
& -\int_{B_{2}} M^{E}(s, x) K_{\theta, s}[G(s, \zeta)] d B(s) \\
& +\int_{B_{2}}^{V^{E}}(s, x) G(s, \zeta) d B(s) \\
& +U(\zeta, x), \quad a \leq \rho \leq b
\end{aligned}
$$

\section{ILLUSTRATIVE EXAMPLES}

Case 1: Green's function of the biharmonic equation for the circular plate problem

A circular plate subjected to a concentrated load as shown in Fig. 3 has been solved by Szilard [12] and Melnikov [13]. The concentrated load is set at the center of the plate in the Szilard's solution. Here, we revisit the same problem to obtain an analytical solution, and then verify the validity of our approach after comparing with Szilard and Melnikov solutions. The equivalence between the Melnikov and present solutions is shown in Appendix 1. The displacement contours of a circular plate subjected to a concentrated load are plotted in Fig. 4. Also, two displacement contours with the source at different angles and radial positions are plotted by using the Melnikov's solution and the present analytical solution as shown in Figs. $5 \sim 6$. Good agreement is made after comparing with the Melnikov's solutions. Although Melnikov offered a closed-form solution in the circular plate, extension to a closed-form solution for an annular plate is not straightforward. Based on our formulation, we can deal with the annular case in a straightforward way as well as a circular plate.

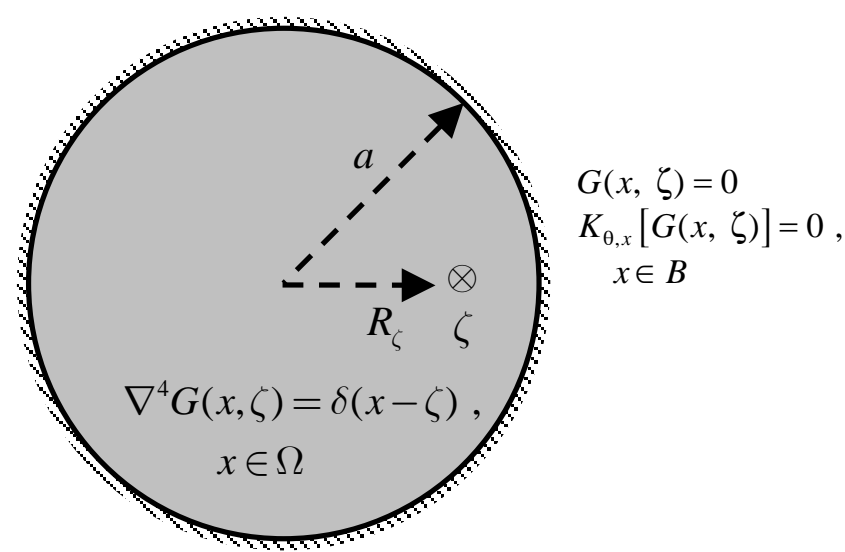

Fig. 3 Green's function of the biharmonic equation for the plate problem
Case 2: Green's function of the biharmonic equation for the fixed-free annular plate problem

For the fixed-free boundary condition, we can obtain the analytical solution from Eq. (37) as shown in Appendix 2. Figure 7 shows the displacement contours of Green's function by using FEM (ABAQUS) and the present method, respectively. Good agreement is obtained between our analytical solution and FEM result. The radial slope, normal moment and shear force contours are shown in Fig 8. Next, we present different cases of boundary conditions.

Case 3: Green's function of the biharmonic equation for the fixed-fixed annular plate problem

For the fixed-fixed annular plate problem, the displacement and the slope on the outer and inner circles are both zero for the fixed boundary. Figure 9(a) is the displacement contour by using the present method and the ABAQUS program. Good agreement is obtained. The analytical solutions had been shown in the Liao's thesis [14]. The convergence property of the present method has been studied in the Wu's thesis [15]. Since the present method is analytical, collocation points are not required. In our experiences for several cases, the fifty terms of Fourier series were used to yield acceptable results which compared well with the ABAQUS data.

Case 4: Green's function of the biharmonic equation for the free-simply supported annular plate problem

For the free-simply supported annular plate problem, the moment and the shear force on the inner circle are zero for the free boundary and the displacement and the moment on the outer circle are zero for the simply supported boundary. Figure 9(b) is the displacement contour by using the present method and the ABAQUS software. Good agreement is obtained. The analytical solutions can be found in the Liao's thesis [14]. The number of nodes and elements in FEM were shown in Fig. 10.

\section{CONCLUDING REMARKS}

For circular and annular plate problems, an analytical approach by using degenerate kernels, null-field integral equation and Fourier series was proposed. The main gain of the present method is that all the improper integrals are avoided when degenerate kernels are used. Once the Fourier coefficients of the unknown boundary densities were determined, the displacement, slope, normal moment and effective shear force of the circular or annular plate can be easily determined by substituting the boundary densities into the boundary integral equations for the domain point. Not only the circular plate but also the annular problems have been solved analytically by using the present method in comparison with available exact solutions and FEM results. The advantage of the present method is an analytical approach for the circular and annular cases free of boundary elements. However, ABAQUS program is based on the numerical method (FEM). The extension to general boundaries is under investigation. 


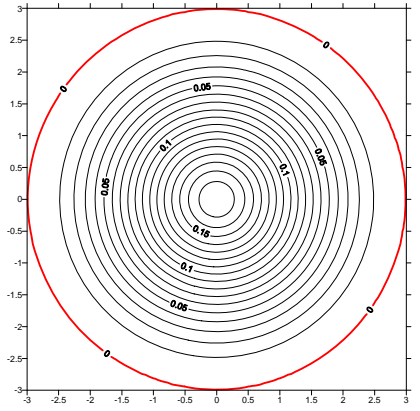

(a) Present method $(M=1)$

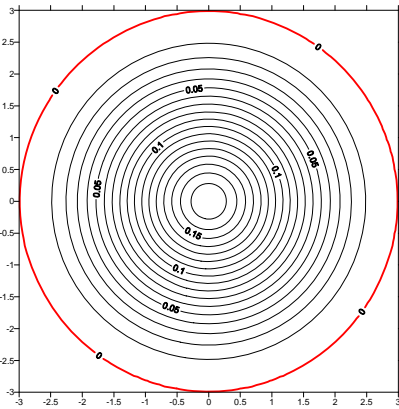

(b) Szilard's approach

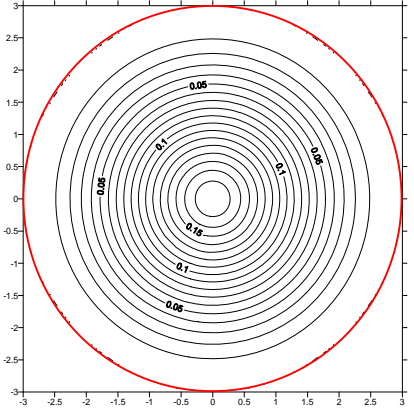

(c) Melnikov's approach

Fig. 4 Displacement contours of the Green's function of the biharmonic equation for the circular plate problem $(a=3)$

Source at different radial angles

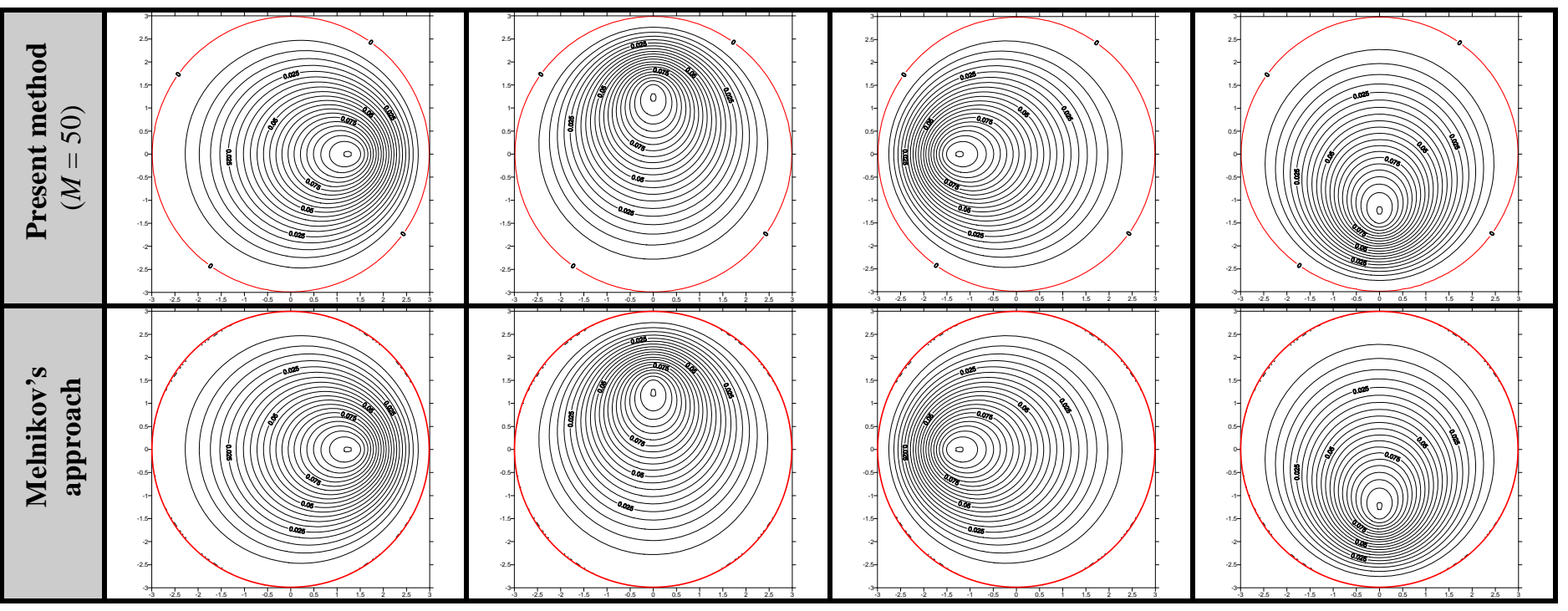
(a) $\theta_{\zeta}=0$
(b) $\theta_{\zeta}=\pi / 2$
(c) $\theta_{\zeta}=\pi$
(d) $\theta_{\zeta}=3 \pi / 2$

Fig. 5 Displacement contours of the Green's function of the biharmonic equation for the circular problem $\left(a=3, R_{\zeta}=1.5\right)$

Source at different radial positions

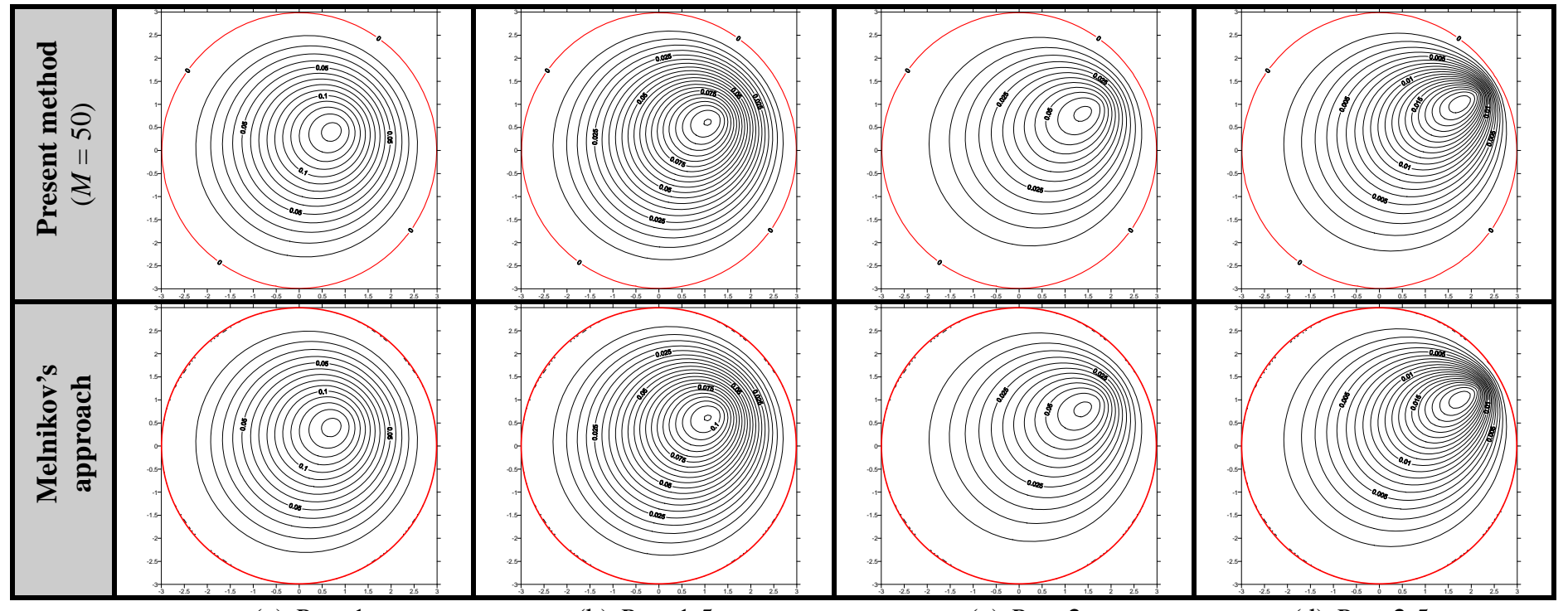

(a) $R_{\zeta}=1$

(b) $R_{\zeta}=1.5$

(c) $R_{\zeta}=2$

(d) $R_{\zeta}=2.5$

Fig. 6 Displacement contours of the Green's function of the biharmonic equation for the circular plate problem $\left(a=3, \theta_{\zeta}=\pi / 6\right)$ 


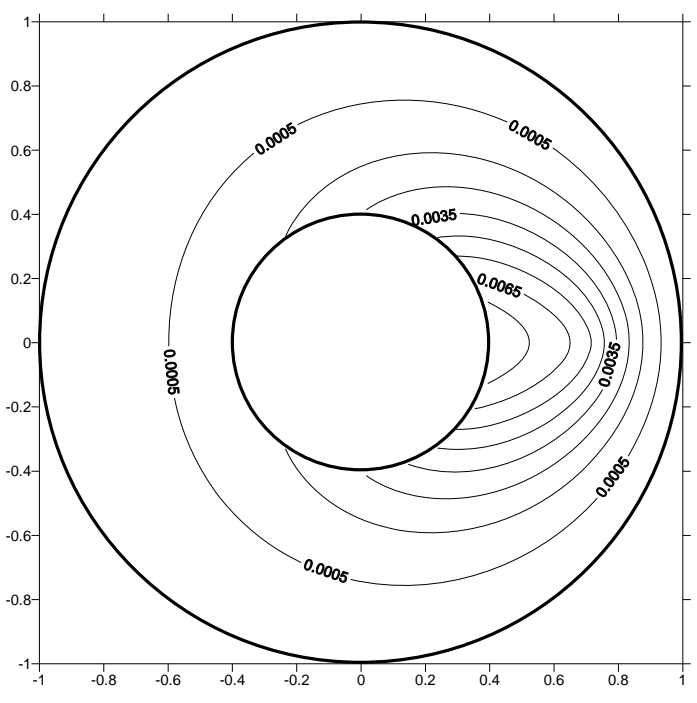

Present method

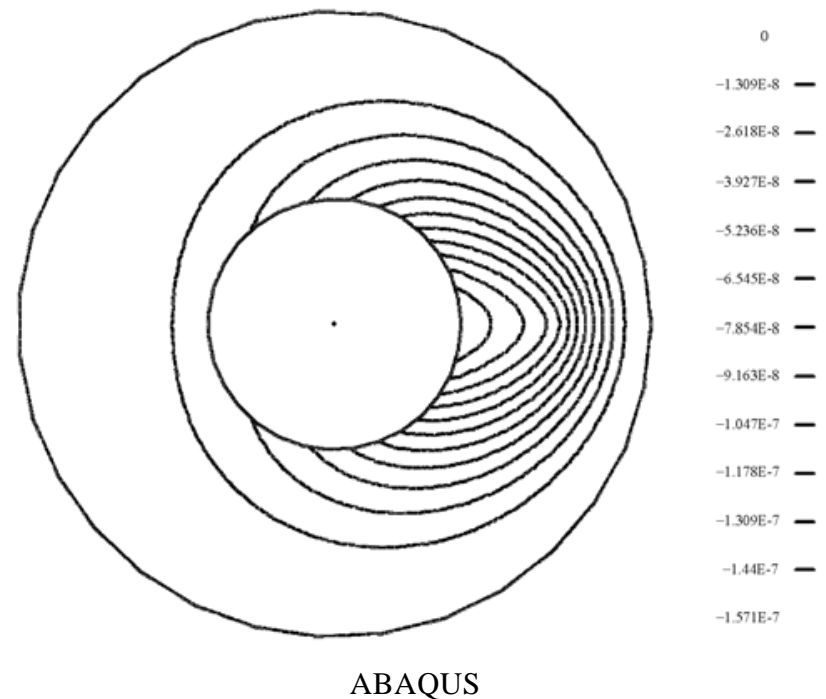

Fig. 7 Displacement contours of the Green's function of the biharmonic equation for the fixed-free plate problem $\left(R_{\zeta}=0.7, \theta_{\zeta}=0, M=50\right)$
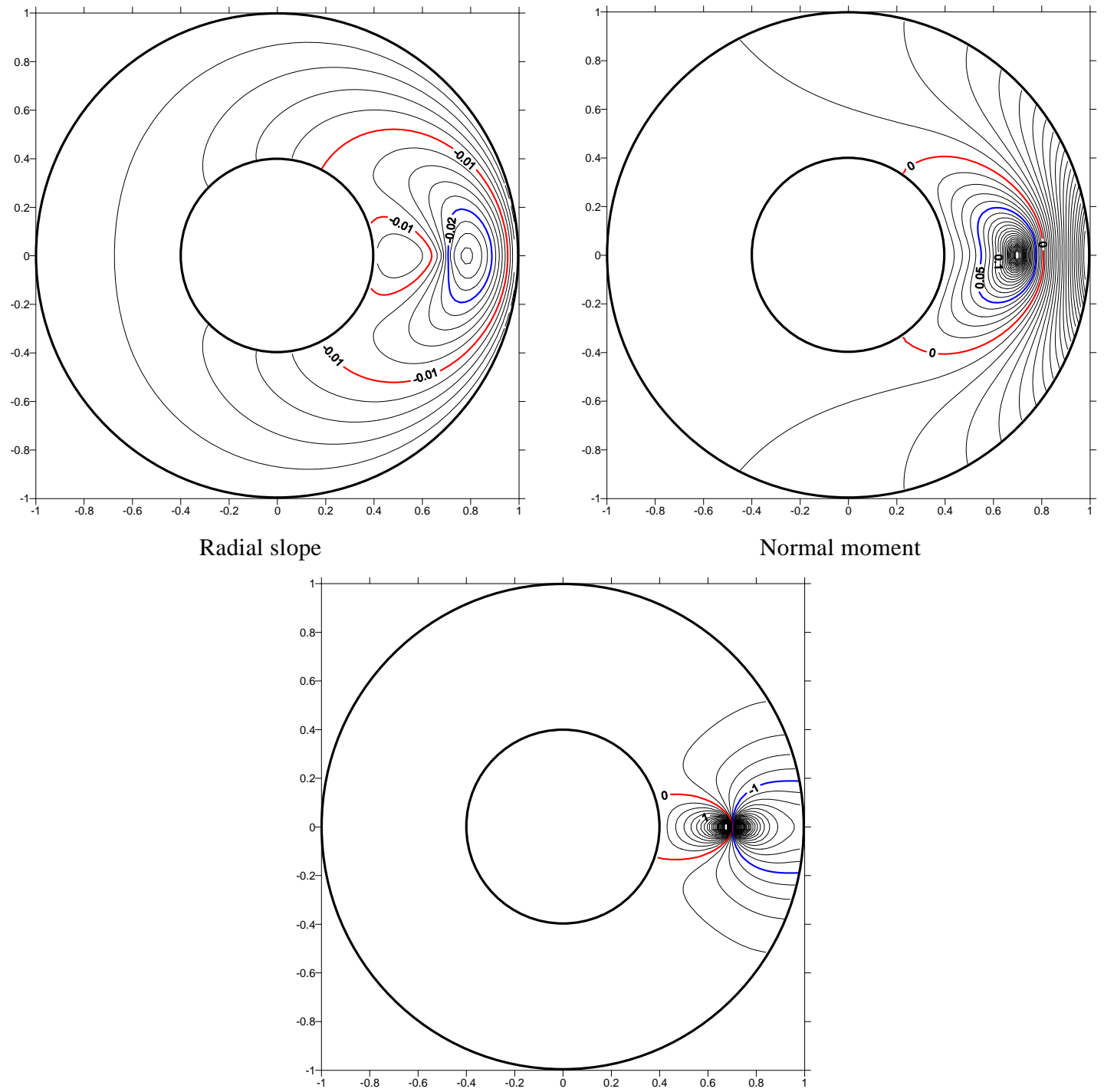

Shear force

Fig. 8 Three contours of the Green's function of the biharmonic equation for the fixed-free annular plate using the present method $\left(R_{\zeta}=0.7, \theta_{\zeta}=0, M=50\right)$ 

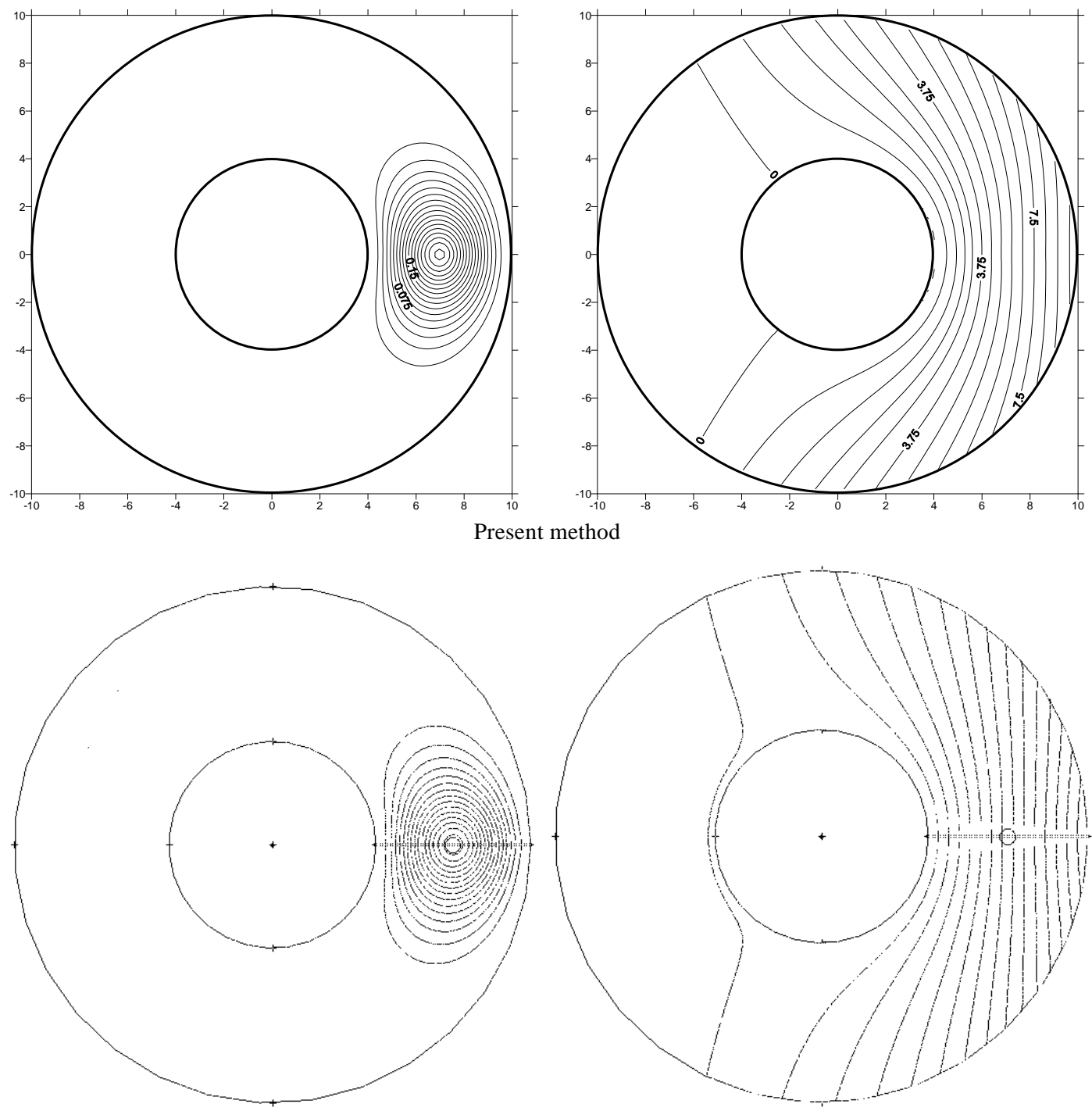

ABAQUS

(a) Fixed-fixed annular plate

(b) Free-simply supported annular plate

Fig. 9 Displacement contours of the Green's function for the biharmonic equation using the present method and the ABAQUS program $\left(R_{\zeta}=0.7, \theta_{\zeta}=0, M=50\right)$

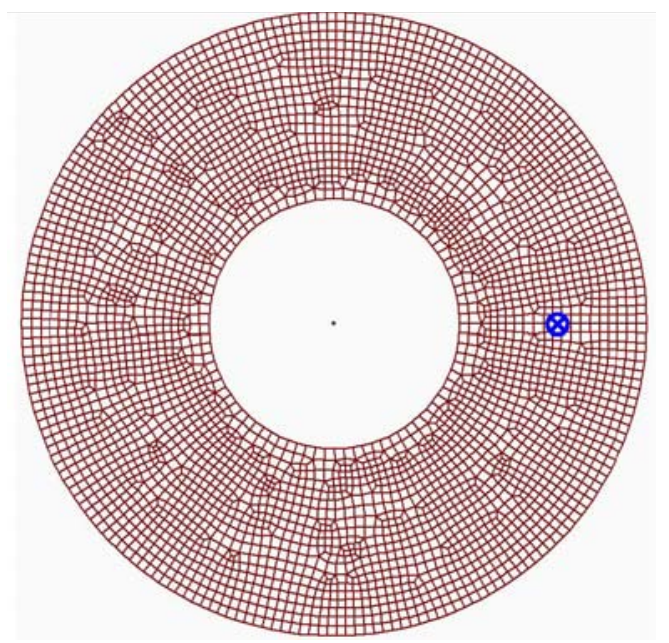

Number of element $=3648$, Number of node $=3794$ The position of the concentrated load $\mathbb{Q}$

Fig.10 The model of FEM figure

\section{APPENDIX 1}

EQUIVALENCE BETWEEN THE PRESENT SOLUTION AND THE MELNIKOV'S RESULT

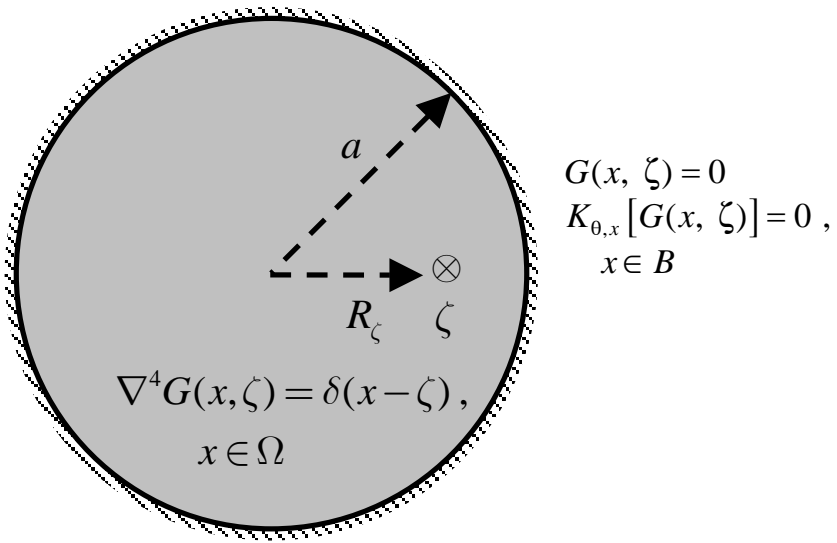

Fig. A-1 Green's function for the biharmonic equation 
For the circular plate subjected to a concentrated load solved by Melnikov [13], the closed-form solution is shown below:

$$
G(x, \zeta)=\frac{1}{8 \pi D}\left[\frac{1}{2 a^{2}}\left(a^{2}-|z|^{2}\right)\left(a^{2}-|\zeta|^{2}\right)-(z-\zeta)^{2} \ln \frac{\left|a^{2}-z \bar{\zeta}\right|}{a|z-\zeta|}\right]
$$

where $D$ is the flexural rigidity, $a$ is the radius, $z=\rho e^{i \phi}$ and $\zeta=R_{\zeta} e^{i \theta \zeta}$ denote the field and the force point, respectively. The problem is also solved by using the present method. We have a series-form solution as shown below:

$$
\begin{aligned}
8 \pi G(x, \zeta)= & -2 \pi a \rho^{2}(1+\ln a) a_{0}-2 \pi a^{3} \ln a a_{0}+\left[a \rho(1+2 \ln a)+\frac{1}{2} \frac{\rho^{3}}{a}\right] \pi a\left(a_{1} \cos \phi+b_{1} \sin \phi\right) \\
& +\sum_{n=2}^{\infty}\left[\frac{1}{n(n+1)} \frac{\rho^{n+2}}{a^{n}}-\frac{1}{n(n-1)} \frac{\rho^{n}}{a^{n-2}}\right] \pi a\left(a_{n} \cos n \phi+b_{n} \sin n \phi\right) \\
& +2 \pi \rho^{2} p_{0}+2 \pi a^{2}(1+2 \ln a) p_{0}-\left[\rho(3+2 \ln a)-\frac{1}{2} \frac{\rho^{3}}{a^{2}}\right] \pi a\left(p_{1} \cos \phi+q_{1} \sin \phi\right) \\
& +\sum_{n=2}^{\infty}\left[\frac{1}{n+1} \frac{\rho^{n+2}}{a^{n+1}}-\frac{n-2}{n(n-1)} \frac{\rho^{n}}{a^{n-1}}\right] \pi a\left(p_{n} \cos n \phi+q_{n} \sin n \phi\right) \\
& +(x-\zeta)^{2} \ln |x-\zeta|, \quad x \in \Omega,
\end{aligned}
$$

where

$$
\begin{gathered}
\left\{\begin{array}{l}
a_{0} \\
p_{0}
\end{array}\right\}=\left\{\begin{array}{c}
\frac{1}{2 \pi a} \\
\frac{a^{2}-R_{\zeta}^{2}}{4 \pi a^{2}}
\end{array}\right\}, \quad\left\{\begin{array}{l}
a_{1} \\
p_{1}
\end{array}\right\}=\left\{\begin{array}{c}
\frac{-\cos \theta_{\zeta} R_{\zeta}\left(R_{\zeta}^{2}-3 a^{2}\right)}{2 \pi a^{4}} \\
\frac{\cos \theta_{\zeta} R_{\zeta}\left(a^{2}-R_{\zeta}^{2}\right)}{2 \pi a^{3}}
\end{array}\right\}, \\
\left\{\begin{array}{l}
a_{n} \\
p_{n}
\end{array}\right\}=\left\{\begin{array}{c}
\frac{a^{-n-3} \cos n \theta_{\zeta} R_{\zeta}^{n}\left[(n+2) a^{2}-n R_{\zeta}^{2}\right]}{2 \pi} \\
q_{1}
\end{array}\right\}=\left\{\begin{array}{c}
\frac{-\sin \theta_{\zeta} R_{\zeta}\left(R_{\zeta}^{2}-3 a^{2}\right)}{2 \pi a^{4}} \\
\left.\frac{\sin \theta_{\zeta} R_{\zeta}\left(a^{2}-R_{\zeta}^{2}\right)}{2 \pi a^{3}}\right\}, \\
\frac{a^{-n-2} \cos n \theta_{\zeta} R_{\zeta}^{n}\left[a^{2}-R_{\zeta}^{2}\right]}{2 \pi}
\end{array}\right\}, \quad\left\{\begin{array}{l}
b_{n} \\
q_{n}
\end{array}\right\}=\left\{\begin{array}{c}
\frac{a^{-n-3} \sin n \theta_{\zeta} R_{\zeta}^{n}\left[(n+2) a^{2}-n R_{\zeta}^{2}\right]}{2 \pi} \\
\frac{a^{-n-2} \sin n \theta_{\zeta} R_{\zeta}^{n}\left[a^{2}-R_{\zeta}^{2}\right]}{2 \pi}
\end{array}\right\} .
\end{gathered}
$$

By rearranging Eq. (A1-1), we have

$$
G(x, \zeta)=\frac{1}{8 \pi D}\left[\frac{1}{2 a^{2}}\left(a^{2}-\rho^{2}\right)\left(a^{2}-R_{\zeta}^{2}\right)-\left[\rho^{2}+R_{\zeta}^{2}-2 \rho R_{\zeta} \cos \left(\theta_{\zeta}-\phi\right)\right]\left[\ln \left|1-\frac{z \bar{\zeta}}{a^{2}}\right|+\ln a\right]+(x-\zeta)^{2} \ln |x-\zeta|\right]
$$

where $\ln \left|1-\frac{x \bar{\zeta}}{a^{2}}\right|$ can be expanded by using the degenerate kernel as shown below:

$$
\ln \left|1-\frac{x \bar{\zeta}}{a^{2}}\right|=-\sum_{m=1}^{\infty} \frac{1}{m}\left(\frac{\rho R_{\zeta}}{a^{2}}\right)^{m} \cos m\left(\theta_{\zeta}-\phi\right), a^{2}>\rho R_{\zeta}
$$

After substituting Eq. (A1-4) into Eq. (A1-3), we can have another series-form solution as shown below:

$$
\begin{aligned}
G(x, \zeta)= & \frac{1}{8 \pi D}\left[\frac{\left(a^{2}-\rho^{2}\right)\left(a^{2}-R_{\zeta}^{2}\right)}{2 a^{2}}-\left(\rho^{2}+R_{\zeta}^{2}\right) \ln a+2 \rho R_{\zeta} \ln a \cos \left(\theta_{\zeta}-\phi\right)\right. \\
& +\sum_{m=1}^{\infty} \frac{\rho^{2}+R_{\zeta}^{2}}{m}\left(\frac{\rho R_{\zeta}}{a^{2}}\right)^{m} \cos m\left(\theta_{s}-\phi\right)-\sum_{m=1}^{\infty} \frac{\rho R_{\zeta}}{m}\left(\frac{\rho R_{\zeta}}{a^{2}}\right)^{m} \cos (m+1)\left(\theta_{\zeta}-\phi\right) \\
& \left.-\sum_{m=1}^{\infty} \frac{\rho R_{\zeta}}{m}\left(\frac{\rho R_{\zeta}}{a^{2}}\right)^{m} \cos (m-1)\left(\theta_{\zeta}-\phi\right)+(x-\zeta)^{2} \ln |x-\zeta|\right],
\end{aligned}
$$


Eq. (A1-5) can be rearranged to

$$
\begin{aligned}
G(x, \zeta)= & \frac{1}{8 \pi D}\left[\frac{\left(a^{2}-\rho^{2}\right)\left(a^{2}-R_{\zeta}^{2}\right)}{2 a^{2}}-\left(\rho^{2}+R_{\zeta}^{2}\right) \ln a-\rho R_{\zeta} \frac{\rho R_{\zeta}}{a^{2}}\right. \\
& +\left[2 \rho R_{\zeta} \ln a+\left(\rho^{2}+R_{\zeta}^{2}\right) \frac{\rho R_{\zeta}}{a^{2}}-\frac{\rho R_{\zeta}}{2}\left(\frac{\rho R_{\zeta}}{a^{2}}\right)^{2}\right] \cos \left(\theta_{\zeta}-\phi\right) \\
& +\sum_{m=2}^{\infty}\left[\frac{\rho^{2}+R_{\zeta}^{2}}{m}\left(\frac{\rho R_{\zeta}}{a^{2}}\right)^{m}-\frac{\rho R_{\zeta}}{m-1}\left(\frac{\rho R_{\zeta}}{a^{2}}\right)^{m-1}-\frac{\rho R_{\zeta}}{m+1}\left(\frac{\rho R_{\zeta}}{a^{2}}\right)^{m+1}\right] \cos m\left(\theta_{\zeta}-\phi\right) \\
& \left.+(x-\zeta)^{2} \ln |x-\zeta|\right],
\end{aligned}
$$

By substituting the Fourier coefficients into Eq. (A1-2), we have

$$
\begin{aligned}
8 \pi G(x, \zeta)=- & \rho^{2}(1+\ln a)-a^{2} \ln a+\rho^{2} \frac{a^{2}-R_{\zeta}^{2}}{2 a^{2}}+(1+2 \ln a) \frac{a^{2}-R_{\zeta}^{2}}{2} \\
& +\left[a \rho(1+2 \ln a)+\frac{1}{2} \frac{\rho^{3}}{a}\right] \frac{R_{\zeta}\left(3 a^{2}-R_{\zeta}^{2}\right)}{2 a^{3}} \cos \left(\theta_{\zeta}-\phi\right) \\
& -\left[\rho(3+2 \ln a)-\frac{1}{2} \frac{\rho^{3}}{a^{2}}\right] \frac{R_{\zeta}\left(a^{2}-R_{\zeta}^{2}\right)}{2 a^{2}} \cos \left(\theta_{\zeta}-\phi\right) \\
& +\sum_{n=2}^{\infty}\left[\frac{1}{n(n+1)} \frac{\rho^{n+2}}{a^{n}}-\frac{1}{n(n-1)} \frac{\rho^{n}}{a^{n-2}}\right] \frac{a^{-n-2} R_{\zeta}^{n}\left[(n+2) a^{2}-n R_{\zeta}^{2}\right]}{2} \cos n\left(\theta_{\zeta}-\phi\right) \\
& +\sum_{n=2}^{\infty}\left[\frac{1}{n+1} \frac{\rho^{n+2}}{a^{n+1}}-\frac{n-2}{n(n-1)} \frac{\rho^{n}}{a^{n-1}}\right] \frac{a^{-n-1} R_{\zeta}^{n}\left[a^{2}-R_{\zeta}^{2}\right]}{2} \cos n\left(\theta_{\zeta}-\phi\right)
\end{aligned}
$$

\begin{tabular}{|c|c|c|}
\hline & Melnikov term & Present term \\
\hline constant & $\frac{\left(a^{2}-\rho^{2}\right)\left(a^{2}-R_{\zeta}^{2}\right)}{2 a^{2}}-\left(\rho^{2}+R_{\zeta}^{2}\right) \ln a-\rho R_{\zeta} \frac{\rho R_{\zeta}}{a^{2}} \equiv$ & $-\rho^{2}(1+\ln a)-a^{2} \ln a+\rho^{2} \frac{a^{2}-R_{\zeta}^{2}}{2 a^{2}}+(1+2 \ln a) \frac{a^{2}-R_{\zeta}^{2}}{2}$ \\
\hline $\cos \left(\theta_{\zeta}-\phi\right)$ & {$\left[2 \rho R_{\zeta} \ln a+\left(\rho^{2}+R_{\zeta}^{2}\right) \frac{\rho R_{\zeta}}{a^{2}}-\frac{\rho R_{\zeta}}{2}\left(\frac{\rho R_{\zeta}}{a^{2}}\right)^{2}\right] \cos \left(\theta_{\zeta}-\phi\right)$} & $\begin{aligned} & {\left[a \rho(1+2 \ln a)+\frac{1}{2} \frac{\rho^{3}}{a}\right] \frac{R_{\zeta}\left(3 a^{2}-R_{\zeta}^{2}\right)}{2 a^{3}} \cos \left(\theta_{\zeta}-\phi\right) } \\
\bar{\equiv} & -\left[\rho(3+2 \ln a)-\frac{1}{2} \frac{\rho^{3}}{a^{2}}\right] \frac{R_{\zeta}\left(a^{2}-R_{\zeta}^{2}\right)}{2 a^{2}} \cos \left(\theta_{\zeta}-\phi\right)\end{aligned}$ \\
\hline $\operatorname{cosm}\left(\theta_{\zeta}-\phi\right)$ & $\begin{aligned} \sum_{m=2}^{\infty}[ & \frac{\rho^{2}+R_{\zeta}^{2}}{m}\left(\frac{\rho R_{\zeta}}{a^{2}}\right)^{m}-\frac{\rho R_{\zeta}}{m-1}\left(\frac{\rho R_{\zeta}}{a^{2}}\right)^{m-1} \\
& \left.-\frac{\rho R_{\zeta}}{m+1}\left(\frac{\rho R_{\zeta}}{a^{2}}\right)^{m+1}\right] \cos m\left(\theta_{\zeta}-\phi\right)\end{aligned}$ & $\begin{aligned} \sum_{n=2}^{\infty}[ & \left.\frac{1}{n(n+1)} \frac{\rho^{n+2}}{a^{n}}-\frac{1}{n(n-1)} \frac{\rho^{n}}{a^{n-2}}\right] \\
\bar{\equiv} & \cdot \frac{a^{-n-2} R_{\zeta}^{n}\left[(n+2) a^{2}-n R_{\zeta}^{2}\right]}{2} \cos n\left(\theta_{\zeta}-\phi\right) \\
& +\sum_{n=2}^{\infty}\left[\frac{1}{n+1} \frac{\rho^{n+2}}{a^{n+1}}-\frac{n-2}{n(n-1)} \frac{\rho^{n}}{a^{n-1}}\right] \\
& \cdot \frac{a^{-n-1} R_{\zeta}^{n}\left[a^{2}-R_{\zeta}^{2}\right]}{2} \cos n\left(\theta_{\zeta}-\phi\right)\end{aligned}$ \\
\hline
\end{tabular}

Although Eqs. (A1-6) and (A1-7) look different, we use Mathematica symbolic software to mathematically prove the equivalence between the two solutions in three aspects, constant, $\cos \left(\theta_{\zeta}-\phi\right)$ and $\cos m\left(\theta_{\zeta}-\phi\right)$ terms as shown in Table A1.

Table A1 Comparison of the Melnikov solution and our solution in three aspects 
$8 \pi G(\zeta, x)=U(\zeta, x)$

$$
\begin{aligned}
& -2 \pi b\left[\rho^{2}(1+\ln b)+b^{2} \ln b\right] a_{0}+2 \pi b\left[\frac{\rho^{2}}{b}+b(1+2 \ln b)\right] \overline{a_{0}}-2 \pi a[2(1+v)(1+\ln \rho)] p_{0} \\
& +\left[b \rho(1+2 \ln b)+\frac{1}{2} \frac{\rho^{3}}{b}\right] \pi b\left(a_{1} \cos \phi+b_{1} \sin \phi\right)-\left[\rho(3+2 \ln b)-\frac{1}{2} \frac{\rho^{3}}{b^{2}}\right] \pi b\left(\overline{a_{1}} \cos \phi+\overline{b_{1}} \sin \phi\right) \\
& +\left[(v+3) \frac{a}{\rho}\right] \pi a\left(p_{1} \cos \phi+q_{1} \sin \phi\right)+(-3-v) \frac{1}{\rho} \pi a\left(\overline{p_{1}} \cos \phi+\overline{q_{1}} \sin \phi\right) \\
& +\sum_{m=2}^{\infty}\left[\frac{1}{m(m+1)} \frac{\rho^{m+2}}{b^{m}}-\frac{1}{m(m-1)} \frac{\rho^{m}}{b^{m-2}}\right] \pi b\left(a_{m} \cos m \phi+b_{m} \sin m \phi\right) \\
& +\sum_{m=2}^{\infty}\left[\frac{1}{m+1} \frac{\rho^{m+2}}{b^{m+1}}-\frac{m-2}{m(m-1)} \frac{\rho^{m}}{b^{m-1}}\right] \pi b\left(\overline{a_{m}} \cos m \phi+\overline{b_{m}} \sin m \phi\right) \\
& -\sum_{m=2}^{\infty}\left[\frac{m(v-1)-2(v+1)}{m} \frac{a^{m}}{\rho^{m}}+(1-v) \frac{a^{m-2}}{\rho^{m-2}}\right] \pi a\left(p_{m} \cos m \phi+q_{m} \sin m \phi\right) \\
& +\sum_{m=2}^{\infty}\left[(m(1-v)-4) \frac{a^{m-1}}{\rho^{m}}-m(1-v) \frac{a^{m-3}}{\rho^{m-2}}\right] \pi a\left(\overline{p_{m}} \cos m \phi+\overline{q_{m}} \sin m \phi\right), \quad a \leq \rho \leq b
\end{aligned}
$$

where

$$
\begin{aligned}
& a_{0}=\frac{1}{2 b \pi} \\
& \bar{a}_{0}=-\left(b^{2}(v-1)+2 a^{2}(v+1)\left(\ln b+\ln R_{\zeta}\right)-(v-1) R_{\zeta}^{2}\right) /\left(4 \pi\left(-b^{2}(v-1)+a^{2}(v+1)\right)\right) \\
& p_{0}=\left(a\left(b^{2}\left(-1+2 \ln b-2 \ln R_{\zeta}\right)+R_{\zeta}^{2}\right)\right) /\left(4 \pi\left(-b^{2}(-1+v)+a^{2}(1+v)\right)\right) \\
& \bar{p}_{0}=\left(\frac{2 a^{2}(1+v)\left(\left(a^{2}-b^{2}\right)+2 b^{2}(\ln b-\ln a)\right)\left(1+\ln R_{\zeta}\right)}{-b^{2}(-1+v)+a^{2}(1+v)}+\frac{\left(\left(b^{2}-a^{2}\right)(-1+v)+2 a^{2}(1+v)(\ln a-\ln b)\right)\left(b^{2}(1+2 \ln b)+R_{\zeta}^{2}\right)}{-b^{2}(-1+v)+a^{2}(1+v)}\right. \\
&\left.+2\left(b^{2} \ln b+(1+\ln b) R_{\zeta}^{2}\right)-2 a^{2}\left(1+\ln R_{\zeta}\right)+R_{\zeta}^{2} \ln R_{\zeta}\right) / 16 \pi \\
& a_{1}=-\left(\left(a^{4} b^{2}(3+v)+\left(-3 b^{4}(-1+v)+a^{4}(3+v)\right) R_{\zeta}^{2}+b^{2}(-1+v) R_{\zeta}^{4}\right)\right) \cos \theta_{\zeta} /\left(2 b^{2} \pi\left(b^{4}(-1+v)-a^{4}(3+v)\right) R_{\zeta}\right) \\
& \bar{a}_{1}=\left(\left(b^{2}-R_{\zeta}^{2}\right)\left(-a^{4}(3+v)+b^{2}(-1+v) R_{\zeta}^{2}\right)\right) \cos \theta_{\zeta} /\left(2 b \pi\left(b^{4}(-1+v)-a^{4}(3+v)\right) R_{\zeta}\right) \\
& p_{1}=-\left(\left(-a^{2} b^{2}\left(-b^{2}(-3+v)+a^{2}(3+v)\right)+\left(-2 a^{2} b^{2}(-3+v)+b^{4}(-1+v)+a^{4}(3+v)\right.\right.\right. \\
&+\left.\left.\left.2\left(-b^{4}(-1+v)+a^{4}(3+v)\right)\left(\ln b-\ln R_{\zeta}\right)\right) R_{\zeta}^{2}+\left(a^{2}(-3+v)-b^{2}(-1+v)\right) R_{\zeta}^{4}\right)\right) \cos \theta_{\zeta} \\
& /\left(8 \pi\left(-b^{4}(-1+v)+a^{4}(3+v)\right) R_{\zeta}\right) \\
& \bar{p}_{1}=\left(a \left(a^{2} b^{2}\left(-b^{2}(1+v)+a^{2}(3+v)\right)+\left(-(a-b)(a+b)\left(-b^{2}(-1+v)+a^{2}(3+v)\right)\right.\right.\right. \\
&\left.\left.\left.+2\left(-b^{4}(-1+v)+a^{4}(3+v)\right)\left(-\ln b+\ln R_{\zeta}\right)\right) R_{\zeta}^{2}-\left(-b^{2}(-1+v)+a^{2}(1+v)\right) R_{\zeta}^{4}\right)\right) \cos \theta_{\zeta} \\
& /\left(8 \pi\left(-b^{4}(-1+v)+a^{4}(3+v)\right) R_{\zeta}\right)
\end{aligned}
$$




$$
\begin{aligned}
b_{1}= & -\left(a^{4} b^{2}(3+v)+\left(-3 b^{4}(-1+v)+a^{4}(3+v)\right) R_{\zeta}^{2}+b^{2}(-1+v) R_{\zeta}^{4}\right) \sin \theta_{\zeta} /\left(2 b^{2} \pi\left(b^{4}(-1+v)-a^{4}(3+v)\right) R_{\zeta}\right) \\
\bar{b}_{1}= & \left(\left(b^{2}-R_{\zeta}^{2}\right)\left(-a^{4}(3+v)+b^{2}(-1+v) R_{\zeta}^{2}\right)\right) \sin \theta_{\zeta} /\left(2 b \pi\left(b^{4}(-1+v)-a^{4}(3+v)\right) R_{\zeta}\right) \\
q_{1}= & -\left(\left(-a^{2} b^{2}\left(-b^{2}(-3+v)+a^{2}(3+v)\right)+\left(-2 a^{2} b^{2}(-3+v)+b^{4}(-1+v)\right.\right.\right. \\
& \left.+a^{4}(3+v)+2\left(-b^{4}(-1+v)+a^{4}(3+v)\right)\left(\ln b-\ln R_{\zeta}\right)\right) R_{\zeta}^{2}+\left(a^{2}(-3+v)\right. \\
& \left.\left.\left.-b^{2}(-1+v)\right) R_{\zeta}^{4}\right)\right) \sin \theta_{\zeta} /\left(8 \pi\left(-b^{4}(-1+v)+a^{4}(3+v)\right) R_{\zeta}\right) \\
\bar{q}_{1}= & \left(a \left(a^{2} b^{2}\left(-b^{2}(1+v)+a^{2}(3+v)\right)+\left(-(a-b)(a+b)\left(-b^{2}(-1+v)\right.\right.\right.\right. \\
& \left.\left.+a^{2}(3+v)\right)+2\left(-b^{4}(-1+v)+a^{4}(3+v)\right)\left(-\ln b+\ln R_{\zeta}\right)\right) R_{\zeta}^{2} \\
& \left.\left.-\left(-b^{2}(-1+v)+a^{2}(1+v)\right) R_{\zeta}^{4}\right)\right) \sin \theta_{\zeta} /\left(8 \pi\left(-b^{4}(-1+v)+a^{4}(3+v)\right) R_{\zeta}\right) \\
& \left.+a^{2 n} b^{2 n}\left(b^{4} n^{2}(-1+v)^{2}-2 a^{2} b^{2}\left(-1+n^{2}\right)(-1+v)^{2}+a^{4}\left(n^{2}(-1+v)^{2}+8(1+v)\right)\right)\right) \\
a_{n}= & \left(b ^ { - 1 + n } R _ { \zeta } ^ { - n } \left(a ^ { 2 ( 1 + n ) } \left(a^{2 n} b^{2}(-2+n)(-1+v)(3+v)+b^{2 n}\left(-b^{2}\left(-2+n+n^{2}\right)(-1+v)^{2}\right.\right.\right.\right. \\
& \left.\left.+a^{2}\left(n^{2}(-1+v)^{2}+8(1+v)\right)\right)\right)+a^{2 n} n(-1+v)\left(b^{2 n}\left(-a^{2}(1+n)+b^{2}(2+n)\right)(-1+v)\right. \\
& \left.-a^{2(1+n)}(3+v)\right) R_{\zeta}^{2}+R_{\zeta}^{2 n}\left(-a^{2} b^{2 n}(-1+v)(3+v)\left(b^{2}(2+n)-n R_{\zeta}^{2}\right)\right. \\
& +a^{2 n}\left(a ^ { 2 } \left(-b^{2}(-2+n)(1+n)(-1+v)^{2}+a^{2}\left(n^{2}(-1+v)^{2}\right.\right.\right. \\
& \left.\left.\left.\left.+8(1+v)))-\left(-b^{2}(-2+n)+a^{2}(-1+n)\right) n(-1+v)^{2} R_{\zeta}^{2}\right)\right)\right)\right) \cos n \theta_{\zeta} \\
& /\left(2 \pi \left(-a^{2+4 n} b^{2}(-1+v)(3+v)-a^{2} b^{2+4 n}(-1+v)(3+v)\right.\right. \\
& \\
&
\end{aligned}
$$

$$
\begin{aligned}
\bar{a}_{n}= & -\left(b ^ { n } R _ { \zeta } ^ { - n } \left(a ^ { 2 ( 1 + n ) } \left(a^{2 n} b^{2} n(-1+v)(3+v)+b^{2 n}\left(b^{2}(-1+n) n(-1+v)^{2}\right.\right.\right.\right. \\
& \left.\left.-a^{2}\left(n^{2}(-1+v)^{2}+8(1+v)\right)\right)\right)+a^{2 n} n(-1+v)\left(b^{2 n}\left(-b^{2} n+a^{2}(1+n)\right)(-1+v)\right. \\
& \left.-a^{2(1+n)}(3+v)\right) R_{\zeta}^{2}+R_{\zeta}^{2 n}\left(a^{2} b^{2 n} n(-1+v)(3+v)\left(b^{2}-R_{\zeta}^{2}\right)\right. \\
& +a^{2 n}\left(a^{2}\left(-b^{2} n(1+n)(-1+v)^{2}+a^{2}\left(n^{2}(-1+v)^{2}+8(1+v)\right)\right)-n\left(a^{2}(-1+n)\right.\right. \\
& \left.\left.\left.\left.\left.-b^{2} n\right)(-1+v)^{2} R_{\zeta}^{2}\right)\right)\right)\right) \cos n \theta_{\zeta} /\left(2 n \pi \left(-a^{2+4 n} b^{2}(-1+v)(3+v)\right.\right. \\
& -a^{2} b^{2+4 n}(-1+v)(3+v)+a^{2 n} b^{2 n}\left(b^{4} n^{2}(-1+v)^{2}-2 a^{2} b^{2}\left(-1+n^{2}\right)(-1+v)^{2}\right. \\
& \left.\left.\left.+a^{4}\left(n^{2}(-1+v)^{2}+8(1+v)\right)\right)\right)\right)
\end{aligned}
$$




$$
\begin{aligned}
p_{n}= & \left(a ^ { 1 + n } R _ { \zeta } ^ { - n } \left(b ^ { 2 ( 1 + n ) } ( - 1 + n ) \left(-a^{2} b^{2 n}(-4+n(-1+v))\right.\right.\right. \\
& \left.+a^{2 n}\left(a^{2}(1+n)(4+n(-1+v))-b^{2} n^{2}(-1+v)\right)\right)+b^{2 n} n(1+n)\left(b^{2(1+n)}\right. \\
& \left.(-1+v)+a^{2 n}\left(b^{2}(-1+n)(-1+v)+a^{2}(-4+n-n v)\right)\right) R_{\zeta}^{2} \\
& +R_{\zeta}^{2 n}\left(b ^ { 2 } ( 1 + n ) \left(a^{2(1+n)}(4+n(-1+v))+b^{2 n}\left(a^{2}(-1+n)(-4+n(-1+v))\right.\right.\right. \\
& \left.\left.-b^{2} n^{2}(-1+v)\right)\right)+(-1+n) n\left(-a^{2 n} b^{2}(-1+v)+b^{2 n}\left(b^{2}(1+n)(-1+v)\right.\right. \\
& \left.\left.\left.\left.\left.+a^{2}(4+n-n v)\right)\right) R_{\zeta}^{2}\right)\right)\right) \cos n \theta_{\zeta} /\left(2 ( - 1 + n ) n ( 1 + n ) \pi \left(-a^{2+4 n} b^{2}(-1+v)\right.\right. \\
& (3+v)-a^{2} b^{2+4 n}(-1+v)(3+v)+a^{2 n} b^{2 n}\left(b^{4} n^{2}(-1+v)^{2}-2 a^{2} b^{2}\left(-1+n^{2}\right)\right. \\
& \left.\left.\left.(-1+v)^{2}+a^{4}\left(n^{2}(-1+v)^{2}+8(1+v)\right)\right)\right)\right)
\end{aligned}
$$

$$
\begin{aligned}
\bar{p}_{n}= & \left(a ^ { 2 + n } R _ { \zeta } ^ { - n } \left(b ^ { 2 ( 1 + n ) } ( - 1 + n ) \left(a^{2} b^{2 n}(2-n+(2+n) v)\right.\right.\right. \\
& \left.+a^{2 n}\left(-b^{2} n^{2}(-1+v)+a^{2}(1+n)(n(-1+v)-2(1+v))\right)\right) \\
& +b^{2 n} n(1+n)\left(-b^{2(1+n)}(-1+v)+a^{2 n}\left(b^{2}(-1+n)(-1+v)\right.\right. \\
& \left.\left.+a^{2}(2+n+2 v-n v)\right)\right) R_{\zeta}^{2}+R_{\zeta}^{2 n}\left(b ^ { 2 } ( 1 + n ) \left(a^{2(1+n)}(n(-1+v)\right.\right. \\
& \left.-2(1+v))+b^{2 n}\left(b^{2} n^{2}(-1+v)-a^{2}(-1+n)(2-n+(2+n) v)\right)\right) \\
& +(-1+n) n\left(-a^{2 n} b^{2}(-1+v)+b^{2 n}\left(-b^{2}(1+n)(-1+v)+a^{2}(2-n\right.\right. \\
& \left.\left.\left.+(2+n) v))) R_{\zeta}^{2}\right)\right)\right) \cos n \theta_{\zeta} /\left(2 ( 1 - n ) n ^ { 2 } ( 1 + n ) \pi \left(-a^{2+4 n} b^{2}(-1+v)(3+v)\right.\right. \\
& -a^{2} b^{2+4 n}(-1+v)(3+v)+a^{2 n} b^{2 n}\left(b^{4} n^{2}(-1+v)^{2}-2 a^{2} b^{2}\left(-1+n^{2}\right)\right. \\
& \left.\left.\left.(-1+v)^{2}+a^{4}\left(n^{2}(-1+v)^{2}+8(1+v)\right)\right)\right)\right) \\
& \left.\left.\left.+2 a^{2} b^{2}\left(-1+n^{2}\right)(-1+v)^{2}+a^{4}\left(n^{2}(-1+v)^{2}+8(1+v)\right)\right)\right)\right) \\
b_{n}= & b^{-1+n} R_{\zeta}^{-n}\left(a ^ { 2 ( 1 + n ) } \left(a^{2 n} b^{2}(-2+n)(-1+v)(3+v)+b^{2 n}\left(-b^{2}\left(-2+n+n^{2}\right)(-1+v)^{2}\right.\right.\right. \\
& \left.\left.+a^{2}\left(n^{2}(-1+v)^{2}+8(1+v)\right)\right)\right)+a^{2 n} n(-1+v)\left(b ^ { 2 n } \left(-a^{2}(1+n)\right.\right. \\
& \left.\left.+b^{2}(2+n)\right)(-1+v)-a^{2(1+n)}(3+v)\right) R_{\zeta}^{2}+R_{\zeta}^{2 n}\left(-a^{2} b^{2 n}(-1+v)(3+v)\left(b^{2}(2\right.\right. \\
& \left.+n)-n R_{\zeta}^{2}\right)+a^{2 n}\left(a ^ { 2 } \left(-b^{2}(-2+n)(1+n)(-1+v)^{2}+a^{2}\left(n^{2}(-1+v)^{2}\right.\right.\right. \\
& \left.\left.\left.\left.+8(1+v)))-\left(-b^{2}(-2+n)+a^{2}(-1+n)\right) n(-1+v)^{2} R_{\zeta}^{2}\right)\right)\right)\right) \sin n \theta_{\zeta} \\
& /\left(2 \pi \left(-a^{2+4 n} b^{2}(-1+v)(3+v)-a^{2} b^{2+4 n}(-1+v)(3+v)+a^{2 n} b^{2 n}\left(b^{4} n^{2}(-1+v)^{2}\right.\right.\right. \\
& \\
&
\end{aligned}
$$




$$
\begin{aligned}
& \bar{b}_{n}=-\left(b ^ { n } R _ { \zeta } ^ { - n } \left(a ^ { 2 ( 1 + n ) } \left(a^{2 n} b^{2} n(-1+v)(3+v)+b^{2 n}\left(b^{2}(-1+n) n(-1+v)^{2}\right.\right.\right.\right. \\
& \left.\left.-a^{2}\left(n^{2}(-1+v)^{2}+8(1+v)\right)\right)\right)+a^{2 n} n(-1+v)\left(b^{2 n}\left(-b^{2} n+a^{2}(1+n)\right)\right. \\
& \left.(-1+v)-a^{2(1+n)}(3+v)\right) R_{\zeta}^{2}+R_{\zeta}^{2 n}\left(a^{2} b^{2 n} n(-1+v)(3+v)\left(b^{2}-R_{\zeta}^{2}\right)+a^{2 n}\left(a^{2}\right.\right. \\
& \left(-b^{2} n(1+n)(-1+v)^{2}+a^{2}\left(n^{2}(-1+v)^{2}+8(1+v)\right)\right)-n\left(a^{2}(-1+n)\right. \\
& \left.\left.\left.\left.\left.-b^{2} n\right)(-1+v)^{2} R_{\zeta}^{2}\right)\right)\right)\right) \sin n \theta_{\zeta} /\left(2 n \pi \left(-a^{2+4 n} b^{2}(-1+v)(3+v)\right.\right. \\
& -a^{2} b^{2+4 n}(-1+v)(3+v)+a^{2 n} b^{2 n}\left(b^{4} n^{2}(-1+v)^{2}-2 a^{2} b^{2}\left(-1+n^{2}\right)\right. \\
& \left.\left.\left.(-1+v)^{2}+a^{4}\left(n^{2}(-1+v)^{2}+8(1+v)\right)\right)\right)\right) \\
& q_{n}=\left(a ^ { 1 + n } R _ { \zeta } ^ { - n } \left(b ^ { 2 ( 1 + n ) } ( - 1 + n ) \left(-a^{2} b^{2 n}(-4+n(-1+v))\right.\right.\right. \\
& \left.+a^{2 n}\left(a^{2}(1+n)(4+n(-1+v))-b^{2} n^{2}(-1+v)\right)\right)+b^{2 n} n(1+n)\left(b^{2(1+n)}\right. \\
& \left.(-1+v)+a^{2 n}\left(b^{2}(-1+n)(-1+v)+a^{2}(-4+n-n v)\right)\right) R_{\zeta}^{2} \\
& +R_{\zeta}^{2 n}\left(b ^ { 2 } ( 1 + n ) \left(a^{2(1+n)}(4+n(-1+v))+b^{2 n}\left(a^{2}(-1+n)(-4+n(-1+v))\right.\right.\right. \\
& \left.\left.-b^{2} n^{2}(-1+v)\right)\right)+(-1+n) n\left(-a^{2 n} b^{2}(-1+v)+b^{2 n}\left(b^{2}(1+n)(-1+v)\right.\right. \\
& \left.\left.\left.\left.\left.+a^{2}(4+n-n v)\right)\right) R_{\zeta}^{2}\right)\right)\right) \sin n \theta_{\zeta} /\left(2 ( - 1 + n ) n ( 1 + n ) \pi \left(-a^{2+4 n} b^{2}(-1+v)\right.\right. \\
& (3+v)-a^{2} b^{2+4 n}(-1+v)(3+v)+a^{2 n} b^{2 n}\left(b^{4} n^{2}(-1+v)^{2}-2 a^{2} b^{2}\left(-1+n^{2}\right)\right. \\
& \left.\left.\left.(-1+v)^{2}+a^{4}\left(n^{2}(-1+v)^{2}+8(1+v)\right)\right)\right)\right) \\
& \bar{q}_{n}=\left(a ^ { 2 + n } R _ { \zeta } ^ { - n } \left(b ^ { 2 ( 1 + n ) } ( - 1 + n ) \left(a^{2} b^{2 n}(2-n+(2+n) v)\right.\right.\right. \\
& \left.+a^{2 n}\left(-b^{2} n^{2}(-1+v)+a^{2}(1+n)(n(-1+v)-2(1+v))\right)\right) \\
& +b^{2 n} n(1+n)\left(-b^{2(1+n)}(-1+v)+a^{2 n}\left(b^{2}(-1+n)(-1+v)\right.\right. \\
& \left.\left.+a^{2}(2+n+2 v-n v)\right)\right) R_{\zeta}^{2}+R_{\zeta}^{2 n}\left(b ^ { 2 } ( 1 + n ) \left(a^{2(1+n)}(n(-1+v)\right.\right. \\
& \left.-2(1+v))+b^{2 n}\left(b^{2} n^{2}(-1+v)-a^{2}(-1+n)(2-n+(2+n) v)\right)\right) \\
& +(-1+n) n\left(-a^{2 n} b^{2}(-1+v)+b^{2 n}\left(-b^{2}(1+n)(-1+v)+a^{2}(2-n\right.\right. \\
& \left.\left.\left.+(2+n) v))) R_{\zeta}^{2}\right)\right)\right) \sin n \theta_{\zeta} /\left(2 ( 1 - n ) n ^ { 2 } ( 1 + n ) \pi \left(-a^{2+4 n} b^{2}(-1+v)(3+v)\right.\right. \\
& -a^{2} b^{2+4 n}(-1+v)(3+v)+a^{2 n} b^{2 n}\left(b^{4} n^{2}(-1+v)^{2}-2 a^{2} b^{2}\left(-1+n^{2}\right)\right. \\
& \left.\left.\left.(-1+v)^{2}+a^{4}\left(n^{2}(-1+v)^{2}+8(1+v)\right)\right)\right)\right)
\end{aligned}
$$

where $n=2,3,4 \cdots$

\section{ACKNOWLEDGEMENTS}

Financial Support from National Science Council under Grant No. NSC-97-2211-E-019-015-MY3 and MOE-CMBB-97-G-A-601 is gratefully acknowledged.

\section{REFERENCES}

1. Boley, B. A., “A Method for the Construction of
Green's Functions,” The Quarterly Journal of Mechanics and Applied Mathematics, 14, pp. 249-257 (1956).

2. Wang, X. and Sudak, L. J., "Antiplane TimeHarmonic Green's Functions for a Circular Inhomogeneity with an Imperfect Interface,” Mechanics Research Communications, 34, pp. 352-358 (2007).

3. Timoshenko, S. and Woinowsky-Krieger, S., The- 
ory of Plates and Shells, McGraw-Hill, New York (1959).

4. Melnikov, Y. A. and Melnikov, M. Y., “Green’s Function for Mixed Boundary Value Problems in Regions of Irregular Shape," Electronic Journal of Boundary Elements, 4, pp. 82-104 (2006).

5. Melnikov, Y. A. and Sheremet, V. D., "Some New Results on the Bending of a Circular Plate Subject to a Transverse Point Force," Mathematics and Mechanics of Solids, 6, pp. 29-45 (2001).

6. Melnikov, Y. A., "Influence Functions of a Point Source for Perforated Compound Plates with Facial Convection," Journal of Engineering Mathematics, 49, pp. 253-270 (2004).

7. Sharafutdinov, G. Z., "Stress and Concentrated Forces in Thin Annular Plates,” Journal of Applied Mathematics and Mechanics, 68, pp. 39-51 (2004).

8. Adewale, A. O., "Isotropic Clamped-Free Thin Annular Circular Plate Subjected to a Concentrated Load," Journal of Applied Mechanics, 73, pp. 658-663 (2006).

9. Chen, J. T., Wu, C. S. and Chen, K. H., “A Study of Free Terms for Plate Problems in the Dual Boundary Integral Equations,” Engineering Analysis with Boundary Element, 29, pp. 435-446 (2005).

10. Chen, J. T., Wu, C. S., Chen, K. H. and Lee, Y. T., "Degenerate Scale for the Analysisof Circular Thin Plate Using the Boundary Integral Equation Method and Boundary Element Methods," Computational Mechanics, 38, pp. 33-49 (2006).
11. Chen, J. T., Hsiao, C. C. and Leu, S. Y., “A New Method For Stokes’ Flow with Circular Boundaries Using Degenerate Kernel and Fourier Series," International Journal for Numerical Methods in Engineering, 74, pp. 1955-1987 (2008).

12. Szilard, R., Theory and Analysis of Plates Classical and Numerical Methods, Englewood Cliffs, New Jersey (1974).

13. Melnikov, Y. A., "Influence Functions of a Point Force for Kirchhoff Plates with Rigid Inclusions," Journal of Mechanics, 20, pp. 249-256 (2004).

14. Liao, H. Z., “Analytical Solutions for the Green's Functions of Laplace and Biharmonic Problems with Circular Boundaries,” MS Thesis, National Taiwan Ocean University, Taiwan (2007),

15. Wu, A. C., "Null-Field Approach for Multiple Circular Inclusion Problems in Anti-Plane Piezoelectricity,” MS Thesis, National Taiwan Ocean University, Taiwan (2006).

16. Chen, J. T. and Chen, P. Y., “A Semi-Analytical Approach for Stress Concentration of Cantilever Beams with Holes Under Bending," Journal of Mechanics, 20, pp. 211-221 (2007).

(Manuscript received October 23, 2007, accepted for publication July 19, 2008.) 